\title{
AN EXTENSION OF A RESULT OF LIAPOUNOFF ON THE RANGE OF A VECTOR MEASURE ${ }^{1}$
}

\section{HERMAN CHERNOFF}

Liapounoff ${ }^{2}$ established in 1940 that the range of a countably additive finite measure with values in a finite-dimensional real vector space is bounded and closed and in the nonatomic case convex. A simplified proof of this result was given by Halmos ${ }^{3}$ in 1948. The aim of the present paper is to extend this result to the following case. Let $\mu_{i t}, 1 \leqq i \leqq k, 1 \leqq t \leqq n_{i}$, be a set of countably additive, finite measures. If $\left\{\left(E_{1}, E_{2}, \cdots, E_{k}\right)\right\}$ is the totality of decompositions of a space $X$ into $k$ pairwise disjoint measurable sets, the range $R$ of the vector $\psi$ with components $\mu_{i t}\left(E_{i}\right), i=1,2, \cdots, k, t=1,2, \cdots, n_{i}$, is bounded, closed, and in the nonatomic case convex.

Let $X$ be any set and let $\delta$ be a $\sigma$-field of subsets of $X$ (called the measurable sets of $X$ ). A measure $\mu$ (one-dimensional) is non-negative if $\mu(E) \geqq 0$ for every $E \in S ; \mu^{*}(E)$ will denote the total variation of $\mu$ on $E .^{4}$ The measure $\mu$ is absolutely continuous with respect to the measure $\nu$ if $\mu$ and $\nu$ are defined on $\mathcal{S}$ and $\mu^{*}(E)=0$ for every $E \in \mathcal{S}$ for which $\nu^{*}(E)=0$. A necessary and sufficient condition that $\mu$ be absolutely continuous with respect to $\nu$ is that for every $\epsilon>0$ there exist a $\delta>0$ such that $\mu^{*}(E)<\epsilon$ for all $E \in S$ so that $\nu^{*}(E)<\delta .\left\{E_{i}\right\}$, $i=1,2, \cdots, k$, is said to be a decomposition of $F$ if the $E_{i}$ are pairwise disjoint measurable subsets of $X$ and $U_{i} E_{i}=F$. Let $\mu_{i t}$,

Presented to the Society, November 25, 1950; received by the editors October 20, 1950.

1 The extension of Liapounoff's result was obtained by a different method and previous to the writing of this paper by A. Dvoretzky, A. Wald, and J. Wolfowitz as a by-product of the proof of another theorem. A generalization of this other theorem in the case of finite measures was also obtained by the author before discovering the work of Dvoretzky, Wald, and Wolfowitz. See their papers, Elimination of randomization in certain problems of statistics and of the theory of games, Proc. Nat. Acad. Sci. U.S.A. vol. 36 (1950) pp. 256-259; also, Relations among certain ranges of vector measures, Pacific Journal of Mathematics (1951). Overlapping results have also been obtained by D. Blackwell. See On a theorem of Lyapunov, Ann. Math. Statist. vol. 22 (1951) pp. 112-115 and The range of certain vector integrals, Proceedings of the American Mathematical Society vol. 2 (1951) pp. 390-395.

2 A. Liapounoff, Sur les fonctions vecteurs complètement additives, Bull. Acad. Sci. URSS. Sér. Math. vol. 4 (1940) pp. 465-478.

${ }^{3} \mathrm{P}$. Halmos, The range of a vector measure, Bull. Amer. Math. Soc. vol. 54 (1948) pp. 416-421. (Much of our notation is taken from this paper.)

4 For classical definitions and results of measure theory we refer to S. Saks, Theory of the integral, Warsaw, 1937, and P. Halmos, Measure theory, Nostrand, 1950. 
$i=1,2, \cdots, k, t=1,2, \cdots n_{i}$, be a set of countably additive finite measures defined on $\mathcal{S}$. We define $\psi$ on the decompositions of $X$ as the vector whose components are $\mu_{i t}\left(E_{i}\right), i=1,2, \cdots, k, t=1,2, \cdots, n_{i}$. The range of $\psi$ is $R$.

Note 1 . Since all the $\mu_{i t}$ are obviously absolutely continuous with respect to the non-negative measure $\nu(E)=\sum_{i, t} \mu_{i t}^{*}(E)$, the RadonNikodým theorem permits us to represent the $\mu_{i t}$ as integrals, that is, $\mu_{i t}(E)=\int_{E} f_{i t}(x) d \nu(x)$.

A measurable set $E$ is an atom of a measure $\mu$ if $\mu(E) \neq 0$ and if for every measurable set $F \subset E$ either $\mu(F)=0$ or $\mu(F)=\mu(E)$. A measurable set $E$ is said to be an atom of $\psi$ if the vector $\phi(E)$ whose components are $\mu_{i t}(E), i=1,2, \cdots, k, t=1,2, \cdots, n_{i}$, is not zero and if for every measurable $F \subset E$ either $\phi(F)=\phi(E)$ or $\phi(F)=0 . \psi$ is said to be nonatomic on $F \in S$ if none of the measures $\mu_{i t}$ has an atom on a subset of $F . \psi$ is said to be purely atomic on $F$ if there is a denumerable sequence $\left\{F_{i}\right\}$, where the $F_{i}$ are pairwise disjoint atoms of $\psi$ and $F=U_{i} F_{i}$.

Note 2. It is easy to see that corresponding to any atom $E$ of any of the measures $\mu_{i t}$ there is an atom $F \subset E$ of $\psi$, and that $X$ may be expressed as the union of two disjoint sets $X_{1}, X_{2}$ where $\psi$ is nonatomic on $X_{1}$ and $\psi$ is purely atomic on $X_{2}$.

LeMma 1. If $\psi=\left(\mu_{11}\left(E_{1}\right), \mu_{12}\left(E_{1}\right), \cdots, \mu_{k n_{k}}\left(E_{k}\right)\right)$ is nonatomic on $X$, the range $R$ of $\psi$ is convex.

Proof. Suppose that $\psi=a$ for the decomposition $E_{1}, E_{2}, \cdots, E_{k}$ and $\psi=b$ for the decomposition $F_{1}, F_{2}, \cdots, F_{k}$. Suppose $0 \leqq \lambda \leqq 1$. Consider the vector measure whose components are $\mu_{r t}(E), r=i, j$, $t=1,2, \cdots, n_{r}$, for the measurable subsets $E$ of $E_{i} \cap F_{j}$. By the Liapounoff Theorem the range of this vector measure is convex and hence $E_{i} \cap F_{j}$ may be decomposed into two disjoint measurable sets $V_{i j}, W_{i j}$ so that $\mu_{r t}\left(V_{i j}\right)=\lambda \mu_{r t}\left(E_{i} \cap F_{j}\right), r=i, j, t=1,2, \cdots, n_{r}$, and hence $\mu_{j t}\left(W_{i j}\right)=(1-\lambda) \mu_{j t}\left(E_{i} \cap F_{j}\right), t=1,2, \cdots, n_{j}$. Consider the decomposition $G_{1}, G_{2}, \cdots, G_{k}$ where $G_{i}=\bigcup_{j}\left[V_{i j} \cup W_{j i}\right]$. It is easily seen that for this decomposition $\psi=\lambda a+(1-\lambda) b$.

Our proof that $R$ is closed will consist of showing that any terminal point of the closure of $R$ is in $R .^{\mathbf{5}}$

Lemma 2. For a given set of constants $\alpha_{i t}$, the function of $\psi$, $\sum_{i, t} \alpha_{i t} \mu_{i t}\left(E_{i}\right)$ attains its maximum.

Proof. Let

${ }^{5}$ We use terminal point of a convex set to mean boundary point with respect to the lowest-dimensional hyperplane containing the set. 


$$
\nu_{i}(E)=\sum_{i=1}^{n_{i}} \alpha_{i t} \mu_{i t}(E), \quad g_{i}(x)=\sum_{t=1}^{n_{i}} \alpha_{i t} f_{i t}(x)
$$

Then

$$
\nu_{i}(E)=\int_{E} g_{i}(x) d \nu(x)
$$

Let

$$
\begin{aligned}
& T_{i_{1} i_{2} \cdots i_{r}}=\left\{x: g_{i_{1}}(x)=g_{i_{2}}(x)=\cdots=g_{i_{r}}(x)>g_{j}(x)\right. \\
& \text { for all } \left.j \notin\left\{i_{1}, i_{2}, \cdots, i_{r}\right\}\right\} \text {. }
\end{aligned}
$$

It is easily seen now that not only is this lemma true, but that a necessary and sufficient condition that a decomposition maximize $\sum_{i, t} \alpha_{i t} \mu_{i t}\left(E_{i}\right)$ is that except for a set of $\nu$ measure $0, T_{i_{1} i_{2}} \ldots i_{\text {r }}$ $\subset\left(E_{i_{1}} \cup E_{i_{2}} \cup \ldots \cup E_{i_{r}}\right)$.

In the nonatomic case the closure $\bar{R}$ of the convex set $R$ is convex and a supporting plane $\Pi$ of $\bar{R}$ may be written $\sum_{i, t} \alpha_{i t} x_{i t}=c$ where not all $\alpha_{i t}$ are zero and $c=\sup \left\{\sum_{i, t} \alpha_{i t} \mu_{i t}\left(E_{i}\right)\right\}$. Hence we have the following corollary.

CoROLlaRY 1. If $\psi$ is nonatomic with range $R$ and $\Pi$ is a supporting plane of $\bar{R}, \Pi \cap R \neq 0$.

LEMMA 3. If $\psi$ is nonatomic, every point of $\bar{R} \cap \Pi$ is a limit point of $R \cap \Pi$.

Proof. If $\left\{a_{n}\right\}$ is a sequence of points of $R$ converging to a point $a$ of $\bar{R} \cap \Pi$ and the decomposition corresponding to $a_{n}$ is given by $E_{1 n}, E_{2 n}, \cdots, E_{k n}$, we have $\sum_{i=1}^{k} \nu_{i}\left(E_{i n}\right)=\sum_{i=1}^{k} \int_{E_{i n}} g_{i}(x) d \nu(x) \rightarrow c$ $=\max \left\{\sum_{i, t} \alpha_{i t} \mu_{i t}\left(E_{i}\right)\right\}$. It follows that the $\nu$ measure of the set $\left\{x: x \in T_{i_{1} i_{2}} \ldots i_{\text {r }}\right.$ and $\left.x \notin\left(E_{i_{1} n} \cup \ldots \cup E_{i_{r} n}\right)\right\}$ must approach zero. If the decomposition corresponding to $a_{n}$ is modified to $F_{1 n}, F_{2 n}, \cdots$, $F_{k n}$ by adjusting the elements of the $E_{i n}$ so that $x \in T_{i_{1} i_{2} \ldots i_{r}}$ implies $x \in F_{i_{1} n} \cup F_{i_{2} n} \cup \ldots \cup F_{i_{r} n}$, then $\sum_{i, t} \alpha_{i t} \mu_{i t}\left(F_{i n}\right)=c$ and $\mu_{i t}\left(F_{i n}\right)$ $-\mu_{i t}\left(E_{i n}\right) \rightarrow 0$ which gives us our result.

LEMMA 4. If $\psi$ is nonatomic its range $R$ is closed.

Proof. Through any terminal point of $R$ there is a supporting hyperplane $\Pi$ so that $\sum_{i, t} \alpha_{i} \mu_{i t}$ is not identically constant for all points of $R$. It suffices to show that $R \cap \Pi$ is closed. We shall proceed by induction on $k$ and the number $n$ of non-null measures involved in $\psi$. Case $1 . k=1$. This case is trivial. Case 2. $k>1, n=1$. The closure follows from the Liapounoff theorem for one-dimensional measures. Case 3. $k>1, n>1$. Let $R_{i_{1} i_{2}} \ldots i_{r}$ be the range of $\psi$ on the decomposi- 
tions of $T_{i_{1} i_{2} \ldots i_{r}}$ where $E_{i}=0$ if $i \notin\left\{i_{1}, i_{2}, \ldots, i_{r}\right\}$. It will suffice to show that the $R_{i_{1} i_{2} \ldots i_{r}}$ are closed. The induction establishes this immediately for all sets except $R_{12} \ldots k$. On this set $g_{1}=g_{2}=\ldots$ $=g_{k}$. Corresponding to one of the non-null measures $\mu_{j s}$ there is a nonzero $\alpha_{j \varepsilon}$. By induction the range $R_{12}^{\prime} \ldots k$, of the vector $\psi^{\prime}$ which has all components of $\psi$ except $\mu_{j s}$, is closed. $\mu_{j s}$ is a linear function of the components of $\psi^{\prime}$ since $\sum_{i, t} \alpha_{i t} \mu_{i t}=\int_{T_{1,2}, \ldots, k} g_{1}(x) d \nu(x)$. Hence $R_{12} \ldots k$ is closed.

LemMa 5. If $\psi$ is purely atomic, its range $R$ is closed.

Proof. This proof is an obvious extension of Liapounoff's. Consider the sequence of atoms $\left\{F_{n}\right\}$ of $\psi$. If $E_{i}$ contains $F_{n}$ (except possibly for a null set), let $a_{i n}=2$ and otherwise 0 . Let $a$ $=\left(a_{1}, a_{2}, \cdots, a_{n}\right)$ where $a_{i}=\sum_{n=1}^{\infty} a_{i n} 3^{-n}$. This relation gives a oneto-one correspondence with the decompositions of $X$ (excepting deviations by sets of measure zero) and a bounded closed set of vectors. $\psi$ considered as a function of $a$ is continuous and hence $R$ is closed.

THEOREM 1. The range $R$ of $\psi=\left(\mu_{11}\left(E_{1}\right), \mu_{12}\left(E_{1}\right), \cdots, \mu_{k n_{k}}\left(E_{k}\right)\right)$ on the decompositions of $X$ is bounded and closed and in the nonatomic case convex.

Proof. Lemma 1 gives the convexity, Lemmas 4 and 5 give the closure when considered in connection with Note 2 . The boundedness is trivial because the measures are finite.

COROLlaRY 2. The range of the vector $\Phi=\left(\mu_{11}\left(E_{1}\right), \mu_{12}\left(E_{1}\right), \ldots\right.$, $\left.\mu_{k n_{k}}\left(E_{k}\right)\right)$, where the $E_{i}$ are pairwise disjoint measurable sets, is bounded and closed and in the nonatomic case convex.

Proof. Let $E_{k+1}=X-\bigcup_{i=1}^{k} E_{i}$. The range of $\psi$ on the decompositions $E_{1}, E_{2}, \cdots, E_{k+1}$ has the desired property. The range of $\Phi$ is a projection of the range of $\psi$ and also has the desired property.

A more trivial result would arise in the case where the assumption of disjoint sets is removed.

Let $I$ be the unit interval $(0,1)$, and $m$ the Lebesgue measure on $I$. The measures $\mu_{i t}$ on $X$ may be extended to $\eta_{i t}=\mu_{i t} \times m$ on $X \times I$. Let $\phi$ be the vector whose components are $\eta_{i t}\left(F_{i}\right)$ where $F_{1}, F_{2}, \cdots, F_{k}$ is a decomposition of $X \times I$ into $k$ measurable pairwise disjoint sets.

Theorem 2. The range $H$ of $\phi$ is the convex hull of the range $R$ of $\psi^{6}$

6 This theorem is the generalization of the result of Dvoretzky, Wald, and Wolfowitz referred to in footnote 1 . 
Proof. $H$ is convex because the $\eta_{i t}$ are obviously nonatomic. It is evident that $H \supset R$. Hence it suffices to show that

$$
\sup _{\phi \in H} \sum_{i, t} \alpha_{i t} \eta_{i t} \leqq \sup _{\nu \in R} \sum_{i, t} \alpha_{i t} \mu_{i t} .
$$

Since $\mu_{i t}(E)=\int_{E f_{i t}}(x) d \nu(x), \eta_{i t}(F)=\int_{F f_{i t}}(x, y) d[\nu \times m](x, y)$ where $f_{i t}(x, y)=f_{i t}(x)$. Then $g_{i}(x, y)=\sum_{t} \alpha_{i t} f_{i t}(x, y)=g_{i}(x)$. Hence $\sum_{i, t} \alpha_{i t} \eta_{i t}$ attains its maximum when

$$
\begin{aligned}
\left\{x, y: g_{i_{1}}(x)=\right. & g_{i_{2}}(x)=\cdots=g_{i_{r}}(x)>g_{i}(x) \\
& \text { for all } \left.i \notin\left\{i_{1}, i_{2}, \cdots, i_{r}\right\}\right\} \subset F_{i_{1}} \text { if } i_{1}<i_{2}<\cdots<i_{r} .
\end{aligned}
$$

But this defines a decomposition of $X \times I$ which corresponds to a decomposition of $X$ for which $\sum_{i, t} \alpha_{i t} \mu_{i t}=\sum_{i, t} \alpha_{i t} \eta_{i t}$.

UNIVERSITY OF ILLINOIS 